\title{
Die Entstehung des Lebens
}

\section{Von UlRich Kull, Stuttgart}

Die Evolution der Organismen auf unserer Erde findet eine zureichende Erklärung durch die moderne Evolutionstheorie. Diese geht von den Mutationen und der Selektion, die innerhalb von Populationen wirksam wird, aus. Mit Hilfe dieser Prinzipien allein ist es aber nicht möglich, eine Theorie der Entstehung des Lebens aufzustellen.

Molekularstatistisch betrachtet ist das Leben ein außerordentlich unwahrscheinlicher Prozeß, der dem zweiten Hauptsatz der Thermodynamik (Entropiesatz) zu widersprechen scheint. Dies gilt auch für die Entstehung der informationstragenden und -verarbeitenden, kohärent organisierten und offenen Systeme, die wir als lebende Systeme bezeichnen. Die genetische Substanz der Lebewesen besteht aus Nucleinsäuremolekülen, in deren Basenabfolge die Information enthalten ist. Diese Nucleinsäuremoleküle werden in der Zelle identisch redupliziert. Auf diesem Wege wird die genetische Information bei der Zellteilung weitergegeben. Die Information der Nucleinsäuremoleküle ist in dieser Gestalt für die Zelle nicht ,verwendbar"; sie muß in einem Ubersetzungsmechanismus in eine Form gebracht werden, welche in der Zelle bestimmte Vorgänge verursachen kann. Dieser im Einzelnen sehr komplexe Ubersetzungsmechanismus wird als Transkription und Translation bezeichnet und führt zur Synthese von Proteinen (Eiweißkörpern). Proteine sind als Enzyme die Katalysatoren nahe$\mathrm{zu}$ aller Stoffwechselvorgänge und an der Steuerung aller Lebensvorgänge führend beteiligt. Die Proteine haben aber nicht die Fähigkeit der Reduplikation. Die Struktur der Proteine ist durch die Abfolge der einzelnen Aminosäuren, die zum Protein zusammentreten, vollständig festgelegt. Die Kette von Aminosäuren faltet sich zur richtigen Raumstruktur, die bestimmte Wechselwirkungen mit anderen Molekülen erlaubt und dadurch die Funktion des Proteins ermöglicht.

Von dieser Betrachtung ausgehend kann man auch die Vorgänge der Evolution auf molekularer Ebene verstehen. Zunächst müssen Strukturveränderungen in den Nucleinsäuren, dem genetischen Material der Zelle, eintreten. Diese werden als Mutationen bezeichnet; sie sind Zufallsereignisse. Die Mutationen führen dann zu veränderten Proteinstrukturen. Der Selektionswert der Mutationen ist von ihrer Zufallsentstehung völlig unabhängig (MoNod 1971). Die Selektion als Ausleseprozeß greift am Organismus im Rahmen der Population an, nie an der Nucleinsäure selbst. Sie fungiert als ein Sieb, das aus den zahlreichen Mutationen bestimmte auswählt, die dann innerhalb des Gen-Pools der Population angereichert werden. Die überwiegende Mehrzahl der Mutationen aber verschwindet wieder. 


\section{Älteste Fossilreste}

Stellt man die Frage nach der Entstehung des Lebens, so ist es naheliegend, zunächst die ältesten Fossilreste zu betrachten. Daraus sind Rückschlüsse über die Gestaltung primitivster Lebewesen und über die Zeit ihrer Entstehung möglich. Lange Zeit wurde Corycium enigmaticum aus dem Archaikum Finnlands (Alter ca. 1,5 Milliarden Jahre) als der älteste Fossilrest angesehen. Es handelt sich hierbei um graphitisierte Reste, die aufgrund ihres C-Isotopen-Verhältnisses von Lebewesen, vermutlich Algen, stammen müssen. In den letzten 20 Jahren sind nun eine Reihe von Fundstätten mit teilweise erheblich älteren Lebensresten gefunden worden (vgl. Barghoorn 1971, Glaessner 1971). Die ältesten stammen aus der Fig-Tree-Serie und der darunterliegenden Onverwaacht-Serie des Swazilandsystems in Südafrika. Diese Gesteine besitzen ein Alter von ca. $3,1-3,3$ Milliarden Jahren. Gefunden wurden bakterienartige Formen, die man als Eobacterium isolatum bezeichnet hat, und blaualgenartige Gebilde (Archaeosphaeroides barbertonensis) sowie organisches Material in fädiger Anordnung. In der nordamerikanischen Gunflint-Serie mit einem Alter von etwa 2 Milliarden Jahren sind 12 „Arten“ primitiver Lebewesen nachgewiesen worden, vor allem den Blaualgen zugerechnete Formen. Weiterhin treten in präkambrischen Gesteinen vor ca. 2,6 Milliarden Jahren erstmals Stromatolithen auf, deren Bildung man Blaualgen zuschreiben kann. In der Bitter-Springs-Serie aus Zentralaustralien (Alter ca. 800-900 Millionen Jahre) findet man Gebilde, welche heutigen kugeligen Grünalgen stark ähneln. Es wird behauptet, daß in diesen Resten gelegentlich Zellkerne erhalten seien, womit sie eindeutig als eukaryontisch identifiziert wären. Die Entstehung der Eukaryonten (alle Organismen außer Bakterien und Blaualgen zählen dazu) darf man aufgrund dieser Befunde vor etwa 1200-1500 Millionen Jahren ansetzen. Die ältesten sicher bekannten Metazoen sind ca. 600-650 Millionen Jahre alt (Ediacara-Fauna). Sie waren morphologisch schon hoch entwickelt, so $\mathrm{da} B$ eine längere Phase ihrer Evolution vorhergegangen sein muß.

Faßt man alle diese Ergebnisse der Paläontologie zusammen, so darf man eine biogene Evolution seit mehr als 3 Milliarden Jahren annehmen. Die Entstehung des Lebens muß also vorher erfolgt sein. Davon sind Fossilreste bisher nicht mit Sicherheit bekannt. Die Wahrscheinlichkeit, solche zu finden, ist infolge des hohen Metamorphiegrades der ältesten Gesteine auch nicht groß. Es gibt also keine direkten paläontologischen Hinweise auf die Entstehung des Lebens. Man kann jedoch aus dem Alter der Erde (ca. 4-4,5 Milliarden Jahre) und dem Auftreten der ersten Lebensreste (ca. 3,3 Milliarden, vgl. oben) auf die Zeit der Lebensentstehung schließen. Zur Erklärung des Vorganges selbst stehen nur indirekte Verfahren zur Verfügung. Die Prozesse, welche der Entstehung von Leben vorausgegangen sein müssen, lassen sich gliedern in:

1. die Bildung organischer Verbindungen durch abiogene Synthese;

2. die Bildung von Makromolekülen;

3. die Organisation von Makromolekülen zu morphologischen Einheiten; 
4. Informationserwerb durch diese Einheiten und damit zusammenhängend ihre „Evolution" (prägenetische Evolution).

Diese 4 Stufen sind dabei in erster Linie durch die Art der Vorgänge unterschieden und können durchaus zeitlich nebeneinander abgelaufen sein.

\section{Die Uratmosphäre und die Bildung organischer Verbindungen}

Aus Chemismus und Struktur der - metamorphen - ältesten Sedimentgesteine sowie aus physikochemischen und kosmochemischen Daten lassen sich Schlüsse über die ursprünglichen Verhältnisse auf der Erdoberfläche und in der Atmosphäre ziehen (vgl. Schidsowski 1971). Für die "Uratmosphäre“, die sekundären Ursprungs ist und durch Entgasungsvorgänge entstand, ergibt sich daraus etwa folgendes Bild: Die wichtigsten Bestandteile waren Stickstoff, Wasserstoff (der schon früh in den Weltraum zu entweichen begann), Ammoniak, Wasserdampf, Blausäure, Schwefelwasserstoff, Methan und wahrscheinlich schon von Anbeginn an auch Formaldehyd. Methan, Blausäure und Formaldehyd sind als Kohlenstoff-Quellen für den Aufbau organischen Materials außerordentlich wichtig. Freier Sauerstoff war in der reduzierenden Uratmosphäre nicht vorhanden. Im Wasser der Urozeane gelöst fanden sich Phosphate, Silikate und Metallionen.

$\mathrm{Da}$ es keinen freien Sauerstoff gab, besaß die Atmosphäre keine OzonSchutzschicht. Infolgedessen wurde die Erdoberfläche von einer intensiven UV-Strahlung erreicht. Vom Ozeanwasser wurde diesse kurzwellige Strahlung in den oberflächenahen Schichten (ca. $10 \mathrm{~m}$ ) absorbiert. Die chemischen Verhältnisse waren damals also andersartig. Durch starke und zahlreiche Gewitter, einen vermutlich viel stärkeren Vulkanismus, die UV-Einstrahlung und eine intensivere radioaktive Strahlung (damals waren noch viele kurzlebige Isotope vorhanden, die heute in der Natur nicht mehr vorkommen) war eine ständige Energiezufuhr gewährleistet, die eine fortwährende Bildung einfacher organischer Verbindungen erlaubte. Die Entstehung zahlreicher solcher Stoffe unter simulierten Bedingungen der Uratmosphäre ist experimentell nachgewiesen (vgl. Fox et al. 1970, RABinowITz 1971, Fox 1971).

Die Entstehung von Aminosäuren ist durch die allgemein bekannt gewordenen Versuche von Miller und später auch anderer Autoren erwiesen. Ein Gemisch von Methan, Ammoniak, Wasserstoff und Wasserdampf wird dabei elektrischen Entladungen oder einer starken UV-Strahlung ausgesetzt und man erhält ein Gemisch verschiedener organischer Verbindungen, worin neben zahlreichen Aminosäuren auch mehrere Aldehyde, Blausäure, Ameisen-, Essig-, Glykol- und Milchsäure sowie Harnstoff und andere Amide auftreten. Die einzige Kohlenstoff-Quelle von der ausgegangen wurde, war hier stets Methan. Ein Aminosäurengemisch kann man ferner durch Anwendung von Stoßwellen aus dem erwähnten Ausgangsgemisch erhalten. Bei der Umsetzung von Methan, Ammoniak und Wasserdampf in Gegenwart von Kieselgel als Katalysator bei $1000^{\circ} \mathrm{C}$ und anschließendem Abschrecken des Reaktionsproduktes in ammoniakhaltigem Wasser sind nach Aufarbeitung 13 verschiedene der 20 proteinogenen 
Aminosäuren zu finden, aber nur wenige Aminosäuren, die in heutigen Lebewesen nicht vorkommen. Auch in wässriger Lösung kann man ein Aminosäure-Gemisch erhalten, wenn man Ammoniumsalze mit Formaldehyd unter Einwirkung von UV-Strahlung umsetzt. Voraussetzung für jede Aminosäure-Bildung ist das Vorliegen reduzierender Verhältnisse; es darf also kein freier Sauerstoff vorhanden sein. Schließlich sei erwähnt, daß Aminosäuren in sehr geringen Mengen auch im Mondstaub nachgewiesen worden sind.

Die Bildung von Zuckern erfolgte aus den Vorstufen Formaldehyd und Acetaldehyd. Es ist schon lange bekannt, daß durch deren Kondensation unter dem Einfluß schwacher Basen oder von UV-Licht ein Gemisch verschiedener Zucker entsteht. Beim Erhitzen einer verdünnten Formaldehydlösung mit Kaolinit oder bei Behandlung mit $\gamma$-Strahlung erhält man Ribose und Desoxyribose, die beiden für den Aufbau von Nucleinsäuren erforderlichen Zucker, in merklichen Ausbeuten.

Die Entstehung von Purin- und Pyrimidinbasen, die als Bausteine der Nucleinsäuren auftreten, erfolgte unter Beteiligung von Blausäure. Aus dieser allein kann durch mehrstufige Polymerisation Adenin entstehen. Eine ganz ähnliche Reaktionsfolge führt von Ammoniumcyanid zum Adenin. Auch die Einwirkung energiereicher Elektronenstrahlung auf ein Gemisch von Methan, Ammoniak und Wasser liefert diesen Purinkörper. Für die Bildung einiger anderer Basen ist im Experiment Eisen als Katalysator erforderlich. Dieses kann auf der Ur-Erde als Meteoreisen zur Verfügung gestanden haben. Alle biologisch relevanten Purin- und Pyrimidinbasen sind mittlerweile unter simulierten präbiotischen Bedingungen hergestellt worden.

Die gebildeten Basen können mit Zuckern von Nucleosiden zusammentreten. So liefert eine wässrige Lösung von Adenin und Ribose (bzw. Desoxyribose) unter Einwirkung von UV-Strahlung Adenosin (bzw. Desoxyadenosin) und eine Reihe von Nucleosiden mit anderen Verknüpfungen zwischen Base und Zucker. Unter Beteiligung anorganischer Polyphosphate kommt es auch zur Bildung von Nucleotiden, wobei bevorzugt Mono-, Diund Triphosphate gebildet werden. Die Reaktion zwischen Nucleosiden und $\mathrm{NaH}_{2} \mathrm{PO}$, bei etwa $160^{\circ} \mathrm{C}$ liefert ebenfalls Nucleosidmonophosphate.

Ein wichtiges Problem ist ferner die Entstehung von Energiedonatoren. Diese Funktion hat in den Zellen aller heutigen Lebewesen vor allem das Adenosintriphosphat (ATP) inne. Die ersten Energielieferanten für prämetabolische Reaktionen waren wahrscheinlich anorganische Polyphosphate, welche im Urozean gelöst vorlagen. Im Laufe der Zeit wurden dann organische Phosphatester und Polyphosphate gebildet, die günstiger waren. Eine leicht entstehende Verbindung dieser Art war vermutlich das Cyanogenphosphat<smiles>N#CC(=N)O[Pb]</smiles>

Dieser Stoff kann die Phosphatgruppe auf Zucker übertragen, wobei nur bestimmte Zuckerphosphate gebildet werden. 
Durch Umsetzung von Methan, Ammoniak und Wasser unter der Einwirkung elektrischer Entladungen erfolgt ferner eine Bildung von Porphyrin-Vorstufen und Porphyrinen. Weiterhin ist eine Reaktion von Nucleotiden mit Aminosäuren von Interesse. Dadurch werden unter anderem Aminosäureadenylate gebildet. Von diesen ausgehend findet in wässriger Lösung leicht eine Polymerisation der Aminosäuren statt. Wahrscheinlich haben diese Verbindungen aber vor allem Bedeutung für die Ausbildung der Wechselwirkungen zwischen Polynucleotiden und Aminosäuren bzw. Proteinen (Fox 1971).

Die Hauptmasse der gebildeten organischen Verbindungen lag sicher gelöst im Urozean vor. Diese Lösung organischen Materials wird als „Ursuppe" bezeichnet. Die Konzentration organischer Substanzen wird bis zu $1 \%$ geschätzt, mag aber lokal, etwa in abgeschnürten Küstenbecken, $10 \%$ weit überschritten haben. Im Strandbereich ist ferner mit einer Austrocknung solcher Becken zu rechnen, wodurch Festkörperreaktionen bei Temperaturen von über $100^{\circ} \mathrm{C}$ möglich waren. Zwischen den Verbindungen fanden statistische Reaktionen statt. Dabei wurden auch Polymere gebildet.

\section{Bildung von Makromolekülen}

Die erste Bildung von Polymeren erfolgte auf anderen Wegen als heute bei der Biosynthese in der lebenden Zelle. Im letzteren Fall ist die Bildung der Makromoleküle ein hochgeordneter Prozeß, der an das Vorhandensein von funktionsfähigen Makromolekülen gebunden ist. Die Synthesen von Polymeren im Urozean waren hingegen statistische Reaktionen. Experimentell sind solche Synthesen unter simulierten Bedingungen ebenfalls gelungen. Aus einem Aminosäuregemisch entstehen eiweißartige Verbindungen mit Molekulargewichten zwischen 3000 und über 11000 , die als Proteinoide bezeichnet wurden (S. W. Fox). Man erhält diese Proteinoide entweder bei ca. $150^{\circ} \mathrm{C}$, oder bei Temperaturen unter $100^{\circ} \mathrm{C}$ bei Zusatz anorganischer Polyphosphate in konzentrierter wässriger Lösung. Unter den Aminosäuren des Reaktionsgemisches müssen Asparaginsäure, Glutaminsäure oder Lysin vorhanden sein; alle anderen Komponenten können beliebig gewählt werden. Die Proteinoide besitzen keinen völlig statistischen Aufbau, sondern ihre Zusammensetzung ist von der Reaktionsfähigkeit der einzelnen zur Kondensation gebrachten Aminosäuren abhängig. Proteinoide werden durch einweißabbauende Enzyme langsam abgebaut, was ihren proteinähnlichen Charakter unterstreicht. - Oligopeptide können in wässrigem Milieu auch durch Reaktion freier Aminosäuren in Gegenwart anorganischer Polyphosphate und ferner durch Kondensation verschiedener Cyanamide entstehen.

Entsprechend der Bildung von Proteinoiden aus Aminosäuren ist eine Polymerisation von Nucleotiden zu mehr oder weniger statistisch aufgebauten Polynucleotiden vorstellbar. Im Experiment ist diese Reaktion bisher allerdings nur bei Anwendung von Polyphosphatestern als Energielieferanten gelungen. Durch Schrams wurden auf diesem Wege Polynucleotide mit Molekulargewichten bis 50000 erhalten. Beim Erhitzen von Cytidylsäure in Gegenwart von Polyphosphorsäure auf $65^{\circ} \mathrm{C}$ bilden sich 
Oligocytidylsäuren mit Polymerisationsgraden um 5. Eine Kondensation von Mononucleotiden erfolgt in wäßriger Lösung auch unter dem Einfluß von Cyanamid, sofern Tonmineralien als Katalysatoren zugegen sind.

Es darf als sicher angesehen werden, daß in der Ursuppe neben kleineren organischen Molekülen schon früh Makromoleküle von Eiweiß- und Nucleinsäure-Charakter vorhanden waren. Beide haben sich vermutlich etwa gleichzeitig gebildet. Wenn Polynucleotide vorhanden waren, konnten sie sich bei geeigneter Energiezufuhr auch identisch reduplizieren. Die Replikation erfolgt aufgrund physikochemischer Wechselwirkungen und ist von einer Enzymwirkung unabhängig. Sie hat mit einem Lebensprozeß zunächst nichts zu tun. Lagen Proteinoide in größeren Mengen vor, so trat durch lockere Wechselwirkung zwischen einzelnen Molekülen Aggregation ein.

Die ganze Entstehung von Makromolekülen mußte unter sterilen Bedingungen erfolgen. Solche waren damals naturgemäß gegeben. Heute dagegen bestehen derartige Verhältnisse nirgends auf der Erde. Würden heute geeignete organische Moleküle abiogen gebildet, so würden sie sehr bald von irgendwelchen Organismen "gefressen“. Dies war damals unmöglich; daher konnte die Entwicklung weitergehen.

\section{Entstehung der optisch aktiven Verbindungen}

Viele der in den Lebewesen auftretenden Verbindungen sind optisch aktiv, so z. B. die Zucker und die Aminosäuren. Dabei besteht eine strenge Auswahl der in den Organismen auftretenden optischen Antipoden. So treten in Proteinen nur L-Aminosäuren auf. Diese Stereospezifität der Verbindungen ist im Stoffwechsel der Zellen durch die Enzyme und deren stereospezifischen Aufbau bedingt. Letztlich bleibt also die Frage: wie sind Proteine entstanden, die nur aus L-Aminosäuren aufgebaut sind, oder - noch einfacher formuliert - warum sind D-Aminosäuren in Lebewesen selten und L-Aminosäuren vorherrschend?

Die optisch aktiven Verbindungen müssen bei der Entstehung des Lebens schon vorhanden gewesen sein. Dies geht z. B. daraus hervor, daß die Beziehungen zwischen Protein bzw. Aminosäuren und Nucleinsäuren bzw. Nucleotiden hoch stereospezifisch sind. Optisch aktive Verbindungen sind also während des Ablaufes der chemischen Evolution entstanden. Gegenwärtig gibt es drei ernsthafte Hypothesen über die Entstehung optisch aktiver Stoffe (vgl. HARADA 1970).

1. Zirkular-polarisiertes Licht kann zur Bildung optisch aktiver Verbindungen führen. Das zirkular polarisierte Licht kann aus linear polarisiertem Himmelslicht unter Einfluß des Erdmagnetfeldes gebildet werden. Eine neue Darstellung dieser Hypothese legte MörTBERG (1971) vor.

2. Optisch aktive Verbindungen können an der Oberfläche optisch asymmetrischer Kristalle gebildet werden. Asymmetrische Kristalle kommen in der anorganischen Natur vor; das bekannteste Beispiel ist der Quarz, welcher in zwei enantiomorphen Formen (D- und L-Quarz) auftritt. Nun gibt es aber, statistisch verteilt, gleich viele D- und L-Quarze. Bei statistischen Oberflächenreaktionen müßten sich also die Produkte die 
Waage halten und schließlich ein Racematgemisch liefern. Voraussetzung für die überwiegende Bildung eines optischen Isomeren ist somit eine lokale Anhäufung einer der Quarzformen.

3. Mehrfach wurden Beziehungen der optischen Aktivität zum Gesetz von der Nichterhaltung der Parität angenommen. Dies sei an einem einfachen Beispiel demonstriert: Gegeben sei die Kernzerfallsreaktion

$$
{ }^{\circ 0} \mathrm{CO} \rightarrow{ }^{\circ 0} \mathrm{Ni}+\mathrm{e}^{-}+\nu_{0} .
$$

Die Elektronen, welche bei diesem $\beta$-Zerfall freigesetzt werden, sind polarisiert. Ihre Bremsstrahlung führt zu zirkular-polarisierter $\gamma$-Strahlung, womit Anschluß an Hypothese 1 gewonnen ist. So könnte eine Dissymmetrie auf der Stufe der Elementarteilchen Wirkungen im molekularen Bereich hervorrufen. Nach dieser Ansicht wäre das bevorzugte Auftreten z. B. von L-Aminosäuren durch die Grundgesetze der Materie determiniert.

Wenn sich einmal asymmetrische Proteinoide als primitive Katalysatoren herausgebildet hatten, so fand fortlaufende Verstärkung durch Rückkopplung statt: am Katalysator werden viele neue asymmetrische Moleküle gebildet, dadurch entstehen viele neue asymmetrische Katalysatoren usw.

\section{Organisation der Makromoleküle}

Makromoleküle sind in Wasser höchstens kolloidal löslich. In solchen kolloidalen Lösungen kann es durch Entmischungsvorgänge zur Bildung von diskreten Aggregaten hochmolekularer Verbindungen kommen. Diese können auch kleinmolekulare Verbindungen aus der wäßrigen Lösung einschließen. Man bezeichnet solche Aggregate in der Kolloidchemie als Koazervate. Solche Koazervate kann man, wie OPARIN gezeigt hat, experimentell z. B. aus Protein und Nucleinsäure herstellen. Sie können mikroskopische Tröpfchen bilden, die in der wäßrigen Lösung schweben.

Für die Theorie der Lebensentstehung ist es nun interessant, daß bei Behandlung von Proteinoiden mit heißem Wasser Gebilde mit ganz ähnlichen Eigenschaften und wesentlich höherer Stabilität entstehen. Sie wurden von Fox als Mikrosphären bezeichnet. Diese können infolge der Selbstaggregation von Proteinoiden membranartige Oberflächen ausbilden. Sogar die Bildung von Doppelmembranen (ohne Beteiligung von Lipiden) ist nachgewiesen. Dadurch besitzen diese Mikrosphären osmotische Eigenschaften, außerdem sind ein selektiver Durchtritt von Molekülen durch die Membran und ein „Wachstum“ durch Stoffaufnahme möglich. Die Aggregation von Mikrosphären unter Ausbildung echter Brücken ist ebenfalls gezeigt worden. Ferner sind Sproßungserscheinungen, welche Hefesproßungen morphologisch stark ähneln, nachgewiesen. In präkambrischen $\mathrm{Ge}-$ steinen SW-Afrikas wurden in Quarz eingeschlossen bitumenhaltige Gebilde gefunden, welche experimentell hergestellten Mikrosphären sehr ähnlich sind (MüLLER 1972).

Im Urozean wurden zahlreiche solche morphologische Einheiten gebildet. Von diesen waren manche gegenüber anderen im Vorteil. Begünstigt waren solche, die mehr strukturerhaltende oder -erweiternde Eigenschaften aufwiesen. Dazu gehört vor allem: bessere Fähigkeit zur Erhaltung der 
Oberfläche oder zu ihrer Wiederherstellung nach Beschädigung durch die Selbstaggregation von Proteinoiden, die Ausbildung innerer Membranen zur Trennung verschiedener Reaktionsräume, eine bessere Aufnahmefähigkeit für bestimmte organische Verbindungen des Milieus und eine Bevorzugung einzelner chemischer Vorgänge im Innern der Einheiten. Bei experimentell hergestellten Proteinoiden wurde gezeigt, daß sie als schwache Katalysatoren verschiedener Reaktionen fungieren. Dabei haben Proteinoide unterschiedlicher Zusammensetzung auch verschiedene Aktivität bezüglich der einzelnen Katalysen. Nachgewiesen ist katalytische Funktion bei Hydrolysen, Decarboxylierungs-, Aminierungs- und Desaminierungsreaktionen. Einige dieser Funktionen werden durch Komplexbildung des Proteinoides mit Schwermetallionen gefördert. Proteinoide waren sehr wahrscheinlich die ersten Vorstufen der Enzyme, durch ihre Katalysen wurde in Mikrosphären ein „Proto-Metabolismus“ in Gang gesetzt. Durch die Evolution dieser primitiven Katalysatoren sind dann wirksamere und gleichzeitig spezifischere Enzyme entstanden. Diese Entwicklung, die man als prägenetische Evolution bezeichnen kann, ist durch Selektion hervorgerufen. Eine Selektion durch Umweltbedingungen gibt es also schon von Anbeginn der Evolution an und schon vor der Existenz echter lebender Systeme.

Sicher entstanden auch viele morphologische Einheiten, die Polynucleotide enthielten. Polynucleotide können mit Proteinoiden in Wechselwirkung treten. Dabei ist eine gewisse Spezifität experimentell nachgewiesen (z. B. Nakashima u. Fox 1972), so treten z. B. lysinreiche Protenoide bevorzugt mit Polyuridylsäure zusammen. Diese Spezifität war anfangs natürlich auf das individuelle System beschränkt, da es keine Vererbung und somit keine geordnete Weitergabe von Information gab. Aber Polynucleotide konnten in gewissem Ausmaß steuern, mit welchen der vielen vorhandenen Proteinoide sie sich in einer Einheit aggregierten. Wenn in einem solchen System nun Proteinoide enthalten waren, die primitive katalytische Funktionen von Polynucleotid-synthetisierenden Enzymen aufwiesen, so war die identische Reduplikation von Polynucleotiden erleichtert. Wenn außerdem Proteinoide vorhanden waren, die eine katalytische Wirkung auf die Polymerisation von Aminosäuren ausübten, so konnte dadurch die Synthese neuer Proteinoide besser ablaufen. Sind die genannten Proteinoide mit Katalysefunktionen ihrerseits von der Struktur bestimmter Polynucleotide durch eine - wenn auch zunächst nur schwache - Wechselwirkung abhängig, so kommt es zu einer fortlaufenden Verstärkung durch Rückkopplung; das System schaukelt sich auf und es entstehen selbststreduplizierende Systeme mit einer Weitergabe von Information. Diese kann man als primitivste lebende Systeme auffassen (vgl. das Schema). Aus dieser kurzen Darstellung erkennt man, daß zu Beginn dieser Entwicklung der Informationsfluß noch in beiden Richtungen, vom Polynucleotid zum Proteinoid und umgekehrt, vor sich ging. Erst im Laufe der Zeit wurden die Polynucleotide bzw. Nucleinsäuren aufgrund ihrer Eigenschaften die eigentlichen Informationsträger und die Proteine die Funktionsträger. 


\section{Schema zur prägenetischen Evolution:}

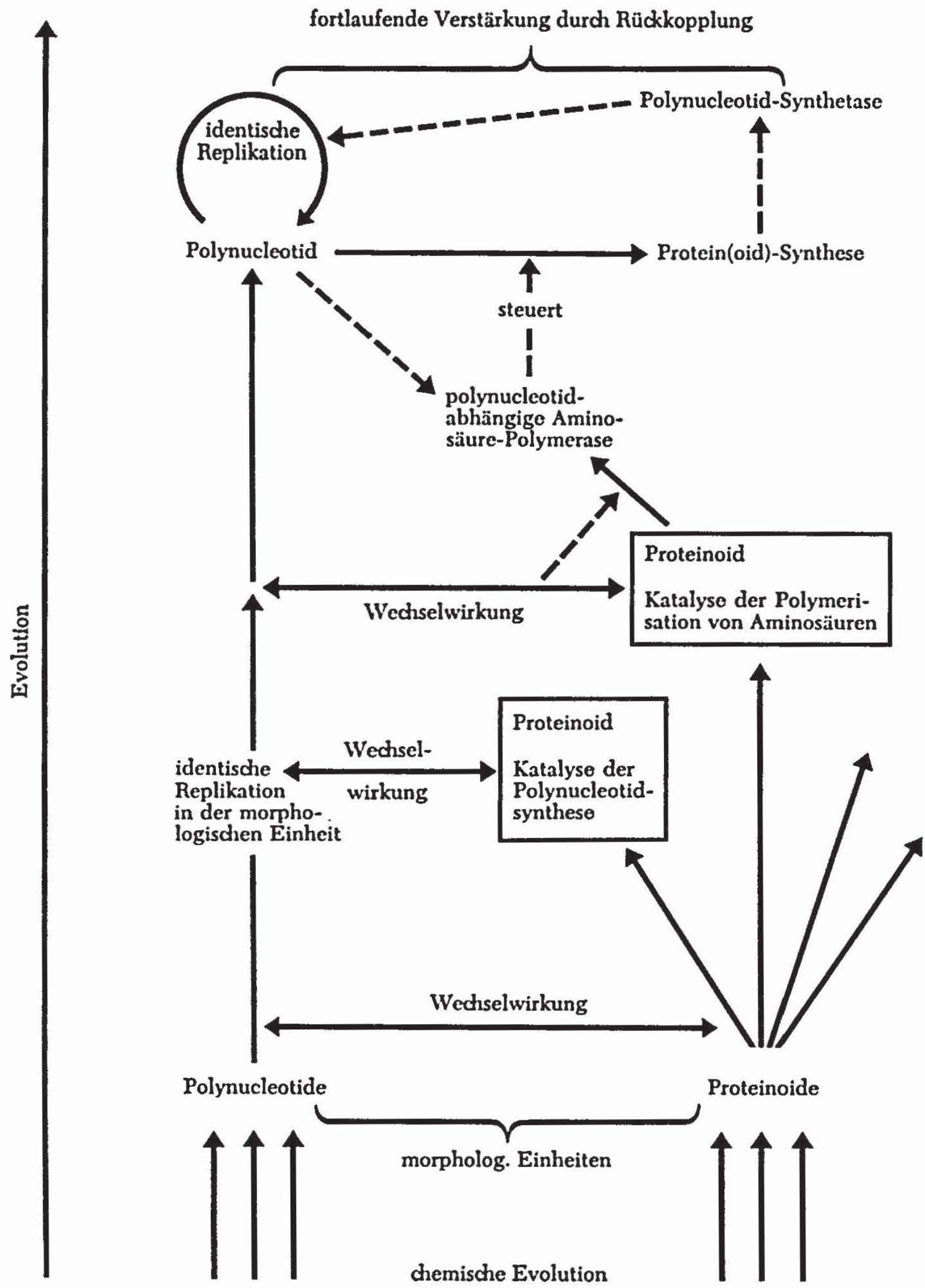


Die Anwendung der stochastischen Kinetik auf diese Evolutionsvorgänge erlaubt nach Eigen (1971, vgl. ScHuster 1972) folgende Schlüsse: die Fähigkeit zur selbstinduzierten Replikation allein reicht aus, um aus einem Gemisch von Informationsträgern, die durch statistische Polymerisation entstanden, wenige Informationsträger "auszuwählen", die übrig bleiben und in der Population herrschend werden. Da die physikalisch-chemischen Eigenschaften der Polymeren gleich sind, kann das Ergebnis des Auswahlvorganges nicht vorhergesagt werden. Es geht auf Fluktuationen zurück, die letztlich auf der quantenmechanischen Unschärfe beruhen. Hier wird das indeterministische Moment der Entstehung des Lebens und des Evolutionsprozesses deutlich.

Eine kurze Ubersicht der Vorgänge, die zur Ausbildung erster Lebewesen, der Eobionten, geführt haben dürften, zeigt das Schema. Die ganze abiogene Evolution bis zu diesen Eobionten war nur möglich, wenn eine große Zahl einzelner morphologischer Einheiten vorhanden war, in welchen dauernd Zufallsveränderungen stattfanden. Schon die präaktualistische Selektion fand also in einer Population von Einheiten statt; sie führte von der chemischen zur biologischen Evolution. Die Selektion ist somit älter als das Leben auf der Erde.

Die Eobionten besaßen die Grundeigenschaften des Lebens: Fähigkeit zur identischen Reproduktion und zu Mutationen sowie Selbstregulation (Homöostase) durch kohärente Organisation. An der Ausbildung der letzteren sind autokatalytische Vorgänge und die Entstehung von Reaktionszyklen erheblich beteiligt (Quispex 1968).

Die Eobionten dürften in flachen Zonen des Ozeans in etwa $10 \mathrm{~m}$ Wassertiefe gelebt haben. Geringere Tiefen wurden von starker UV-Strahlung erreicht und waren „tödlich“. In den Eobionten müssen aus schwach spezifischen Wechselwirkungen die hochspezifischen Vorgänge der Informationsübertragungen der aktualistischen Zellen entstanden sein, so vor allem der genetische Code. Wahrscheinlich waren an dessen Ausbildung primär Wechselwirkungen zwischen Polynucleotiden und Aminoacyladenylaten beteiligt, d.h. molekulare Wechselwirkungen sind als Selektionsfaktoren für die Auswahl der Codewörter anzusehen. Jedoch ist dieser Problemkreis bisher noch ein Feld weiter Spekulationen.

\section{Informationsgewinn}

Die Evolution geht von einer großen Zahl von Zufallsereignissen aus. Die Eobionten aber enthalten Information. Dies führt zu der Frage: wie kommt die Information in die Eobionten hinein? Entscheidende Bedeutung für den Informationsgewinn, während der prägenetischen Evolution ebenso wie im Verlauf der organismischen Evolution, hat der Vorgang der Selektion. Ihre Funktion bei der Entstehung der Eobionten wurde bereits besprochen.

Eine mathematisierte Theorie der Selektion wurde von EIGEN aufgestellt (Ergen 1971, Schuster 1972). Experimente zu einer extrazellulären Evolution von Molekülen aufgrund der Selektion wurden von Spiegelman (1970) mit $Q \beta$ Phagen durchgeführt. - In diesem Zusammenhang muß auch der Ansicht von SchiblowskI (1971), wonach ,die entscheidende qualitative Zäsur, die Urzeu- 


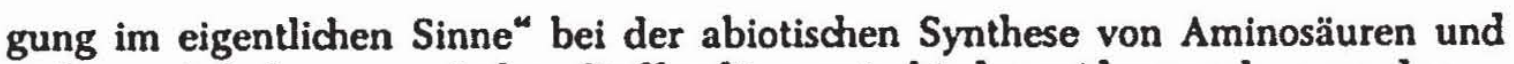
anderen einfachen organischen Stoffen läge, entschieden widersprochen werden.

Information gelangt in das evolvierende System nur, wenn ein Konnex zum Informations-Input der Erdoberfläche (der potentiellen „Biosphäre“) besteht. Dazu wiederum ist ein Zusammenhang zwischen Information und Funktion erforderlich. Das Festhalten von brauchbarer Information geschieht dann durch die Selektion. Eine Funktion haben in den primitiven morphologischen Einheiten zunächst vor allem die Proteinoide. Bei diesen findet zunächst eine Informationszunahme statt. Informations-Input der Erdoberfläche ist in erster Linie die Sonnenstrahlung. Solche Proteinoide, die im Rahmen ihrer katalytischen Funktion einen Verbrauch eines Teils der eingestrahlten Sonnenenergie zu ihrer unmittelbaren Umgebung verursachen, sind der thermischen Zersetzung weniger ausgesetzt und haben somit eine längere mittlere Lebensdauer. In den Einheiten haben diese Proteinoide strukturerhaltende Wirkung und bringen dadurch einen Selektionsvorteil.

Weitere Uberlegungen (ThEodonidis u. Stakx 1971) konnten zeigen, daß der Selektionsvorteil der Proteinoide noch größer ist, wenn die Sonneneinstrahlung periodisch unterbrochen wird, was der natürliche TagNacht-Rhythmus leistet. Die Tagesrhythmik ist also schon für den Informationsgewinn bei der Entstehung von Eobionten von sehr großer Bedeutung. Vielleicht hängt damit die Entstehung der biologischen Uhr (circadiane Rhythmik) als eines Grundphänomens aller lebenden Zellen zusammen.

\section{Evolution der Stoffwechselvorgänge}

In den Eobionten gehen die Evolutionsprozesse als biogene Evolution weiter. Nach der Ausbildung des genetischen Codes und der Ubernahme der Informationsspeicherung auf Nucleinsäuren steht vor allem die Ausbildung von Enzymen und die fortlaufende Erhöhung ihrer Spezifität im Zentrum des Geschehens. Parallel zur Zunahme der Spezifität ist eine Erhöhung der katalytischen Wirksamkeit und damit der Reaktionsgeschwindigkeit der katalysierten Reaktion anzunehmen. Dadurch wird der ursprünglich sehr langsam ablaufende Proto-Metabolismus zu einem echten Stoffwechsel. Neben Proteinoiden hatten anfangs vermutlich auch schon Metallionen katalytische Funktionen. Diese wurden dann in Komplexe eingebaut und letztere schließlich vielfach mit Proteinen kombiniert. So kann man sich die Entstehung erster Enzyme mit Coenzymen vorstellen.

Für die Evolution ist ferner die Vermehrung der Nucleinsäuremenge pro Zelle wichtig. Dadurch kann mehr Information gespeichert werden. Die Vermehrung erfolgt zunächst durch eine Verdoppelung von Nucleinsäureabschnitten infolge einer „Störung“ bei der Replikation. Verdoppelte Stükke unterliegen den zufälligen Mutationen. Sie müssen nicht sofort eine neue Funktion erhalten, sondern können für kürzere oder längere Zeit funktionslos bleiben. Damit findet naturgemäß auch keine Selektion dieser "Gene" statt. Für die Entstehung makromolekularer Komplexität durch derartige Folgeprozesse der Genduplikation hat Woese (1971) ein Modell 
angegeben. Die Bedeutung der Genduplikation für den Evolutionsvorgang wird bei OHNo (1970) ausführlich dargestellt.

Die ersten Lebewesen waren sicher heterotroph; sie ernährten sich vom organischen Material der Ursuppe. Da die Atmosphäre keinen Sauerstoff enthielt, gab es nur einen anaeroben Stoffwechsel. Die Energiegewinnung der Eobionten erfolgte ausschließlich durch Gärungsprozesse. Daran sind sehr wahrscheinlich von Anbeginn Stoffwechselreaktionen der Glykolyse (Embden-Meyerhof-Parnas-Abbau) beteiligt gewesen. Der Kern dieser Reaktionsfolge dürfte ein sehr hohes phylogenetisches Alter aufweisen. Dafür spricht auch sein Vorkommen in allen heutigen Lebewesen, die darauf hin untersucht wurden. Ein ähnlich hohes phylogenetisches Alter dürften Teile des Carbonsäurestoffwechsels haben. Proteinoide sind in diesem Bereich besonders wirksame Katalysatoren (Fox et al. 1970). Der zentrale Teil des Säurestoffwechsels der meisten rezenten Organismen, der Tricarbonsäurezyklus, ist mit der Atmung verknüpft. Dies konnten die ursprünglichen Wege des Säurestoffwechsels natürlich nicht sein. Dagegen sind die von Carbonsäuren ausgehenden Aminierungsreaktionen zu Aminosäuren sicher sehr alt. Als Kern des Ur-Metabolismus darf man somit Reaktionen der Glykolyse, des Säurestoffwechsels und der Aminierung bzw. Transamierung ansehen.

Ein wichtiger Evolutionsschritt war die Erfindung der Photosynthese. Dadurch wurden die Organismen von der langsam abnehmenden Menge organischer Verbindungen des Milieus unabhägig. Durch die Photosynthese kam es zur Bildung von freiem Sauerstoff (vgl. SchidLowski 1971). Dieser wurde anfangs durch Oxidationsvorgänge in der Lithosphäre gebunden, vor allem durch die Oxidation von Eisen. Dadurch war eine allmähliche Anpassung der Organismen an den Sauerstoff möglich. Die ältesten Rotsedimente der Erde, die unter dem Einfluß von Sauerstoff entstanden sein dürften, sind ca. 1,8 Milliarden Jahre alt (CLoud 1968). Schließlich kam es zur Ansammlung freien Sauerstoffs in der Atmosphäre. Lebensformen, die sich dem oxidierenden Milieu nicht angepaßt hatten, wurden infolgedessen vernichtet. So trennt die Erfindung der Photosynthese das ,präaktualistische“ Leben unter reduzierenden Bedingungen vom ,aktualistischen“. Bei der Veränderung der Energiebasis auf der Erdoberfläche hat das Wasser eine Sonderstellung inne. Es ist als einzige der Verbindungen der Uratmosphäre neben dem inerten Stickstoff sowohl im reduzierenden Milieu (als Hydrid des Sauerstoffs) als auch im oxidierenden (als Oxid des Wasserstoffs) stabil. Lebende Systeme, die den Ubergang von präaktualistisch nach aktualistisch erfolgreich vollziehen konnten, mußten also auf der Basis wäßriger Systeme aufgebaut sein. Die Photosynthese ist heute der quantitativ wichtigste biochemische Vorgang überhaupt und liefert durch die $\mathrm{CO}_{2}$-Fixierung letztlich nahezu die gesamte Biomasse der Erde. Die ältesten Wege der Bindung von $\mathrm{CO}_{2}$ standen vermutlich in $\mathrm{Zu}$ sammenhang mit dem Säurestoffwechsel. Solche Reaktionen sind in rezenten Chlorobacteriaceen gefunden worden und können zum reduktiven Carbonsäure-Zyklus zusammengefaßt werden (vgl. KuLL 1971).

Nach der Anhäufung von freiem Sauerstoff wurde ein neuer Mechanismus des Energiegewinns unter Verwendung dieses Sauerstoffs ausgebildet. 
So entstand die Zellatmung. Erforderlich ist dazu ein Sauerstoff-Partialdruck von etwa $1 / 100$ des heutigen Wertes (PASTEur-Punkt). Durch die Verschiebung der Energiebasis wird viel mehr Energie pro abgebautes organisches Molekül verfügbar. Dies war für die Höherentwicklung von Zellkolonien zu echten Vielzellern wahrscheinlich von großer Bedeutung. Die Sauerstoffanreicherung erfolgte zunächst vermutlich in der Nähe von Organismen mit Photosynthese. Daher war dort die Entwicklung auch von atmenden Organismen ohne eigene Photosynthese möglich. Diese waren zunächst an die "Sauerstoff-Oasen" gebunden. Eine isolierte Lage solcher Oasen könnte an der Herausbildung divergierender Evolutionslinien beteiligt gewesen sein. Als der Sauerstoffgehalt der Atmosphäre über $1 \%$ anstieg, wurden die Organismen von den Oasen unabhängig (vor ca. 1,5 bis 1 Milliarden Jahren). Vor etwa 500 Millionen Jahren, vielleicht auch schon früher (vgl. Welte 1970, Glaessner 1971) wurde der 10\%-Spiegel erreicht. Damit war eine Voraussetzung für die Entwicklung von Gefäßpflanzen und Landtieren gegeben.

Sauerstoff ist für die Biosynthese der meisten tierischen Hartteile erforderlich, vor allem für die Bildung von Kollagen, welches seinerseits Voraussetzung jeder Knochenbildung ist. Möglicherweise hängt damit zusammen, daß es im Präkambrium wenige hartschalige Organismen gibt.

\section{Evolution der Zelle}

Der Evolution der Stoffwechselvorgänge parallel läuft eine morphologische Evolution der Zelle unter Ausbildung von Zellorganellen. Die ihrer Organisation nach primitivsten heutigen Organismen sind die Bakterien und Blaualgen. Sie besitzen keinen echten Zellkern, keine Mitochondrien und keine Plastiden. Wegen des Fehlens eines Kernes werden sie als Prokaryonta bezeichnet. Aus solchen Prokaryonten-Zellen haben sich die Zellen mit Kern und den genannten anderen Zellorganellen entwickelt. Diese bezeichnet man als eukaryontische Zellen. Uber die Entstehung des Zellkerns und der Kernteilungsvorgänge ist wenig bekannt. In Organismen von der Bitter-Springs-Fundstelle sollen Mitosevorgänge wahrscheinlich gemacht worden sein.

Die Mitochondrien und Plastiden waren ursprünglich vermutlich selbständige Lebewesen, die durch Endosymbiose in andere Zellen aufgenommen wurden. Mitochondrien dürften aus bakterienartigen Formen mit lebhafter Atmung, Plastiden aus primitiven Blaualgen entstanden sein. Vielleicht sind auch die bei allen Eukaryonten gleichartig aufgebauten GeiBeln und Cilien durch Symbiose, etwa aus einem spirochaetenartigen Bakterium, hervorgegangen. Für diese Hypothese der Entstehung der Eukaryontenzelle durch Symbiose (MARgulis 1970, 1971) sprechen folgende Befunde: Die Plastiden und Mitochondrien sind Organellen sui generis. Sie enthalten Desoxyribonucleinsäure, die ähnliche Strukturen aufweist wie bakterielle DNS. Beide Organellen besitzen eigene Proteinsynthese-Systeme. Die dazu erforderlichen Ribosomen beider Organellen besitzen denselben Aufbau wie Bakterien-Ribosomen, der von demjenigen der Ribosomen des Cytoplasmas der Eukaryontenzelle erheblich abweicht. - Ferner gibt es 
Modelle für solche Symbiosen unter den heutigen Organismen. So ist für das Killer-System von Paramaecium eine endosymbiontische Herkunft nachgewiesen. Die Killer-Faktoren enthalten DNS. Sie sind als frühe Stufen einer endosymbiontischen Integration anzusehen (Gibson et al. 1971). Die Protozoe Cyanophora enthält Blaualgenzellen in Symbiose, wodurch das Tier auch bei Mangel an organischer Nahrung existieren kann. Bei Paramaecium bursaria hat Chlorllea eine entsprechende Funktion. Dabei ist die Zahl der symbiontischen Chlorella-Zellen genetisch reguliert. Bringt man zusätzliche Zellen derselben Chlorella-Art in das Paramaecium ein, so werden sie verdaut. Die Symbiosepartner werden dagegen nie verdaut und bei der Teilung der Paramaecien an die Tochterzellen weitergegeben.

\section{Höherentwicklung}

Nach der Ausbildung von Eukaryonten-Zellen setzte die Höherentwicklung ein, die zur Entstehung von Vielzellern führte. Die individuelle Form verdankt dabei ihren Ursprung Zufallsvorgängen. Ihre Selektion erfolgt in Konkurrenz zu anderen Formen. Der Selektionsprozeß läuft mit Notwendigkeit ab (vgl. Monod 1971). Die Höherentwicklung ist verbunden mit einer Verbesserung der Regulation und Kontrolle innerhalb des Organismus; die Regulationssysteme werden dabei immer komplexer. Höherentwicklung bedeutet eine Informationszunahme, und somit zugleich eine Entropieabnahme. Das scheint dem zweiten Hauptsatz der Thermodynamik zu widersprechen. Die mathematisierte Evolutionstheorie von EIGEN kann zeigen, daß in Wirklichkeit kein Widerspruch vorliegt.

Der zweite Hauptsatz ist eine statistische Aussage. In einem beliebigen makroskopischen System kann lokal, etwa in einem Organismus, im molekularen Bereich eine Entropieabnahme erfolgen. Das wird durch viele andere Orte mit Entropiezunahme überkompensiert. Alle Lebewesen sind offene Systeme mit Fließgleichgewichten. In einem stabilen stationären Zustand, wie er bei bestehendem Fließgleichgewicht vorliegt, strebt die Entropieproduktion einem Minimum zu (Gesetz von Prigogine). Wird nun die Entropieproduktion durch eine statistische Schwankung einmal geringer, so wird der stabile stationäre Zustand verlassen und durch Veränderungen im System ein neuer stabiler Zustand hergestellt. Negative Schwankungen der Entropieproduktion können nach EIGEN durch Mutationen zustande kommen (vgl. SCHUSTER 1972).

Die Mutationen sind Zufallsereignisse, die sich an den Nucleinsäuremolekülen abspielen. Sie bedeuten für die Nucleinsäure selbst gar nichts. Im molekularen Mechanismus der Zelle führen die meisten zu einer Informationsabnahme und damit zu einer Störung. Wenige aber stellen eine Informationsvermehrung dar, eine Verbesserung der kohärenten Organisation. Diese werden durch die Selektion festgehalten und vermehrt, wenn eine anfängliche Phase der Ungewißheit überdauert ist. In dieser Anfangsphase kann durch Zufallsereignisse auch eine Ausmerzung einer positiven Mutante erfolgen. Sobald aber eine bestimmte Zahl von Exemplaren des mutierten Allels überschritten ist, tritt der Verstärkungseffekt ein und die Mutante wird angehäuft. Da die Selektion nie an der Nucleinsäure selbst angreift, die 
Mutation aber stets hier erfolgt, kann eine generelle Höherentwicklung zustande kommen. Die mathematische Formulierung der Selektionstheorie durch Eigen (1971) macht die Selektion durch eine Wertfunktion quantitativ faßbar. Mutanten einer Basensequenz, deren Wertfunktion einen höheren Wert aufweist als bei der ursprünglichen Nucleinsäure, verdrängen die letztere, wenn sie die statistische Anfangsphase überleben. Dieser Optimierungsproze $B$ geht fortlaufend weiter; jedoch können nur Basensequenzen durchprobiert werden, die sich von der jeweils vorherigen durch mögliche Mutationsvorgänge unterscheiden. Es ist also nicht möglich, daß innerhalb eines endlichen Zeitraums beliebige Basensequenzen durchlaufen werden. $\mathrm{Da}$ die Zahl der insgesamt möglichen Basenabfolgen unvorstellbar groß ist, bleibt es völlig unsicher, ob absolute Maximalwerte der Wertfunktion innerhalb endlicher Zeiträume erreicht werden. In der Regel ist dies sogar unwahrscheinlich.

Damit hat das scheinbare physikalische Paradoxon der Höherentwicklung der Organismen eine Erklärung gefunden. Im molekularen Mechanismus, der auf den beiden weitgehend unabhängigen Ebenen der Nucleinsäuren und der Proteine sich abspielt, ist dieses Paradoxon aufgehoben. $\mathrm{Zu}$ seiner Erklärung bedarf es weder der Annahme einer Entelechie noch einer geheimnisvollen Eigenschaft der Materie, die deren Entwicklung determinieren soll (MoNOD 1971).

Die Höherentwicklung führte schließlich zur Entstehung des Menschen. Mit ihm beginnt eine neue Phase der Evolution, da er die Fähigkeit zur Akkumulation erworbener Erfahrungen, zur Ausbildung von Tradition s. l. und damit zur kulturellen Evolution besitzt. Die präbiotische Evolution, welche nach den besprochenen prägenetischen Mechanismen abläuft, führte zur Entstehung von ersten Lebewesen. Die genetische Evolution, die nach den von Darwin erkannten Prinzipien erfolgt, brachte die Höherentwicklung bis zum Menschen zustande. Der Mensch hat durch die Fähigkeit zur Weitergabe erworbener Eigenschaften, die gewissermaßen nach „lamarckistischen" Gesetzmäßigkeiten stattfindet, eine kulturelle Evolution in Gang gesetzt, durch die er sich radikal von allen anderen Lebewesen unterscheidet und seine einmalige Stellung auf der Erde begründet hat.

\section{Schrifttum}

Barghoorn, E. S. (1971): The oldest fossils. Scient. American 224, (5), $30-42$.

Clou d, P. F. (1968): Atmospheric and hydrospheric evolution on the primitive earth. Science 160, 729-736.

Dose, K. (1970): Uber den Ursprung des Lebens. Naturwissensch. 57, $555-560$.

E e ig e n, M. (1971): Selforganization of matter and the evolution of biological macromolecules. Naturwissensch. 58, 465-523.

Fox, S. W., K. Harada, G. Krampitz, and G. Müller (1970): Chemical origin of cells. Chem. Eng. News 48, (26), 80-94.

Fox. S. W. (1971) Chemical origins of cells II. Chem. Eng. News 49, (50), 46-53. 
Gibson, I., M. Chance and J. Williams (1971): Extranuclear DNA and the endosymbionts of Paramecium aurelia. Nature New Biol. 234, 75-77.

Gl a essner, M. F. (1971): Die Entwicklung des Lebens im Präkambrium und seine geologische Bedeutung. Geol. Rundsch. 60, 1323-1339.

Ha rada, K. (1970): Origin and development of optical activity of organic compounds on the primordial earth. Naturwissensch. 57, 114-119.

Ku ll, U. (1971): Neuere Ergebnisse der Photosynthese-Forschung. Jh. Ges. Naturkde. Württemberg 126, 244-255.

M a r gu l i s, L. (1970). Origin of eukaryotic cells. Yale Univ. Press.

- (1971). Symbiosis and evolution. Scient. American 225, (2), 49-57.

Mörtberg, L. (1971): Nonbiotic origin of optical activity. Nature 232, 105-107.

M o n o d, J. (1971): Zufall und Notwendigkeit. Piper-Verlag, München.

M üller, G. (1972): Organic microspheres from the Precambrian of South-West Africa. Nature 235, 90-95.

N a ka shima, R., and S. W. F ox (1972): Selective condensation of aminoacyl adenylates by nucleoproteinoid microparticles. Proc. Nat. Acad. Sci. USA 69, 106-108.

Qu ispel, A. (1968): Pre-biological evolution. Acta Biotheoretica 18, 291-315.

O h n o, S. (1970): Evolution by gene duplication. Springer-Verlag.

Rabinowitz, J. (1971): L'évolution chimique prébiologique. Chimia 25, 54-59.

S chidlowski, M. (1971): Probleme der atmosphärischen Evolution im Präkambrium. Geol. Rundsch. 60, 1351-1384.

S ch u s ter, P. (1972): Vom Makromolekül zur primitiven Zelle - die Entstehung biologischer Funktion. - Chemie in uns. Zeit, 6, 1-16.

S p i e g e I man, S. (1970): Extracellular evolution of replicating molecules. In: F. O. Schmitt (ed.): Neurosciences, Second study program. Rockefeller Univ. Press, New York, 927-945.

T h e od orid is , G. G., and L. S t a rk (1971): Central role of solar information flow in pregenetic evolution. J. theoret. Biol. 31, 377-388.

Welt e, D. H. (1970): Organischer Kohlenstoff und die Entwicklung der Photosynthese auf der Erde. Naturwissensch. 57, 17-23 (1970).

W o e s e, C. R. (1971): Evolution of macromolecular complexity. J. theoret. Biol. $33,29-34$.

Anschrift des Verfassers: Doz. Dr. U. Kull, Biologisches Institut der Universität 7 Stuttgart 60, Ulmer Straße 227. 